\title{
Coronavirus Disease-2019 Clinical Trial Case Studies Within the Biotechnology and Clinical Research Organizations Industry
}

\author{
Melanie K. Pearce, MBA, MPH'; Nathanael E. Hughes, MPH, MPP, MPhil2* \\ 'Brock University, Niagara Region, 1812 Sir Isaac Brock Way, St. Catharines, ON, L2S 3AI, Canada \\ ${ }^{2}$ George Clinical, The Johns Hopkins Bloomberg School of Public Health, Baltimore, MD 2 I 205, USA \\ *Corresponding author \\ Nathanael E. Hughes, MPH, MPP, MPhil \\ Director of Business Development, George Clinical, The Johns Hopkins Bloomberg School of Public Health, Baltimore, MD 2I 205, USA; \\ E-mail: natehughes73@gmail.com
}

\section{Article information}

Received: November 23 ${ }^{\text {rd }}, 2020$; Revised: December 24 ${ }^{\text {th }}, 2020$; Accepted: January 6 ${ }^{\text {th }}, 2021$; Published: January $22^{\text {nd }}$, 2021

\section{Cite this article}

Pearce MK, Hughes NE. Coronavirus disease-2019 clinical trial case studies within the biotechnology and clinical research organizations industry. Clin Trial Pract Open J. 202I; 4(I): 10-12. doi: 10.17I40/CTPOJ-4-II8

\section{INTRODUCTION}

$\mathrm{O}$ March $11^{\text {th }}, 2020$ the World Health Organization (WHO) declared the novel coronavirus disease-2019 (COVID-19) outbreak a global pandemic. Coronaviruses are non-segmented positive-sense ribonucleic acid (RNA) viruses in the Coronaviridae family, which typically affects the respiratory, gastrointestinal, hepatic and neurologic systems. Under Food and Drug Administration (FDA) expanded access programs and federal emergency guidelines, COVID-19 clinical trials have been some of the most successful trials to date. However, significant challenges have been presented for both Clinical Research Organizations (CROs) and the sponsoring companies, as a result of the COVID-19 pandemic. $^{1}$

The focus of this paper is to bring into context the strategic thinking of C-level executives in the biotechnology and CRO industry when faced with a global pandemic and decision-making process considering the critical need of FDA expedited timelines, potential need for expanded access for emergency use, and clinical site expansion to meet the endpoints of the registered clinical study. ${ }^{2}$

This analysis will incorporate two case studies, Humanigen (NASDAQ: HGEN) based in Burlingame, California, USA as well as Durect (NASDAQ: DRRX) in Cupertino, California, USA. Current research being conducted at Humanigen has a focus on acute respiratory distress syndrome (ARDS) while Durect seeks to understand multi-organ failure resulting from COVID-19. ${ }^{3-6}$

Within the larger context of these two case studies, some of the larger, circumscribed issues that CRO's and sponsors (phar- maceutical/biotechnology companies), due to the current volatility of the medical industry, are summarized below:

- Depending on the inclusion/exclusion criteria, when COVID-19 cases began to increase rapidly in early March, there was a concern that CRO's would be competing for COVID-19 intensive care unit (ICU) patients.

- Direct admit from primary care physician (PCP) to hospital or once the patient is in the emergency room (ER) consent for the study

- Challenges of gaining access to the patients due to facility guidelines

- Challenges regarding training the staff on COVID-19 clinical guidelines at ICU, i.e. nurses, rotating shifts, etc.

- Patients journey from point of positive to either testing negative or leaving the facilities or death.

- How to collect data besides objective data rom labs, ventilator setting, vital signs, etc. for an intubated patient.

- Gaining patient consent while on intubation is on and of itself a challenge since an intubated patient cannot provide feedback on their symptoms.

- What percentages of patients leave the ICU alive or dead?

- Pharma/biotech sponsors have often struggled with how to rewrite Investigational New Drug's (IND's) (and eventually write new IND's) for COVID. When it comes to strategic FDA pathways, they often must rely on CRO's. The issue is that CRO's have also had an extremely sharp learning curve during the pandemic.

\section{Statement of Problem}

Since the March 2020 time frame, operation warp speed has picked 
up the pace on vaccines, where both Pfizer and Modern a have released critical efficacy data in their vaccine studies. BNT162b2 is a COVID-19 vaccine candidate developed by BioNTech and Pfizer, and given by intramuscular injection, with $90 \%$ efficacy. While certainly not a panacea, Moderna's messenger ribonucleic acid-1273 (mRNA) Phase 3 trial reported similar efficacy with their vaccine at $94.5 \%{ }^{4-6}$

Below are operational aspects behind two ongoing clinical trials, a Phase 3 and Phase 2 respectively, one of which is in the public domain, a monoclonal antibody for ARDS called Lenzilumab.

\section{HUMANIGEN}

HUMANIGEN considered the data coming out of Wu et al ${ }^{1}$ article that eighty-four patients $(41.8 \%)$ developed ARDS, and of those 84 patients, $44(52.4 \%)$ died. In those who developed ARDS, compared with those who did not, more patients presented with dyspnea.

There is a trend with biotech's submitting an IND for a drug in trial that could potentially be used as a therapeutic agent against ARDS; mAb's and small molecule programs in idiopathic pulmonary fibrosis (IPF) come to mind. That is, a company might have a drug under investigation for another therapeutic indication and will reach out to a CRO to re-write the IND (and eventually submit a new one) for COVID-19. The purpose of this is twofold; one, to create a blue oceans strategy for the company's shareholders where they can have a linchpin on the COVID market as it develops rapidly. Two, submitting an IND for COVID-19 is expeditious because the biotech does not have to write a completely new IND. It is just amended for COVID, and then once approved through expedited timelines, a company can seek expanded access through the FDA pathway. This is what happed with Humanigen. They had submitted an IND to FDA Oncology division for their Phase 1b/2 ZUMA19 CAR-T study (cytokine storm; cytokine release syndromeor cytokine release syndrome (CRS) affects chimeric antigen receptor (CAR)-T patients as does COVID). This was a pre-IND/IND (or PIND) for their Kite CAR-T trial.

At this stage, Humanigen, with the collaboration of a CRO, CTI Clinical Trials, submitted the same IND for review to Infectious Disease experts, John F et al for COVID-19. Eventually there would be a completely new IND for the proposed COVID use. But for the time being, what we did was suggest that Humanigen use their current IND for the 5 completed/ongoing multicentre trial for the prevention of CRS in patients receiving CAR-T for relapsed or refractory cancer (Kite-Gilead trial). CTI successfully received compassionate use/expanded access from the FDA. CTI then started the trial in the USA with 10 proposed sites, and expanded sites to LATAM. Currently, according to Humanigen's corporate website, they have a total of 53 sites including 30 in USA. According to ClinicalTrials.gov the goal is to enrol approximately 300 patients will be randomized to receive lenzilumab+ standard of care (SOC) vs. SOC in a 1:1 ratio. ${ }^{6}$
When awarded the Humanigen Phase 3 trial for ARDS, a press release was sent out to local Bay Area companies and one of them contacted us with a similar interest. They had a program already in a Phase 1 with 2 adult patients, for IPF, and the board decided to submit that IND, also for ARDS COVID patients, and apply the same strategy Humanigen had. In this industry, if you have a template of a deal that worked in the market, as a first-mover advantage (or "blue oceans") strategy, so to speak, you have to immediately iterate and replicate that in the market as soon as you can. That is what the CRO industry administered for COVID IND's in ARDS (CAR-T and IPF).

On November $6^{\text {th }}$, HUMANIGEN announced that the company and the Department of Defense Joint Program Executive Office for Chemical, Biological, Radiological and Nuclear Defense (JPEO-CBRND) have entered into a Cooperative Research and Development Agreement (CRADA) in collaboration with the biomedical advanced research and development authority (BARDA), part of the Office of the assistant secretary for preparedness and response (ASPR) at the US Department of Health and Human Services, to help meet the federal government's Operation Warp Speed goals. The agreement supports development of lenzilumab in advance of a potential Emergency Use Authorization (EUA) for COVID $-19 .^{7}$

\section{DURECT}

Another fascinating trial, also out of the San Francisco area, is being conducted by DURECT. DURECT is currently conducting a double-blind, multi-centre, placebo-controlled, Phase 2 study to evaluate the safety and efficacy of DUR-928 in approximately 80 COVID-19 patients with acute liver or kidney injury to evaluate multi-organ failure in Covid-19 patients in a 3:1 (DUR-928: placebo) ratio. Patients will receive a dose of $150 \mathrm{mg}$ of DUR-928 or placebo by intravenous infusion on day 1 and day 4 in combination with standard of care therapy, which will be determined by the principal investigator (PI) at each clinical trial site. The primary efficacy endpoint is a composite of survival and being free of acute organ failure (free of mechanical ventilation, free of liver failure events and free of renal replacement therapy) at day 28. Patients will be followed for 60-days. Should any drug product be determined by the FDA to be safe and effective for the treatment of COVID-19 while the trial is ongoing, such treatments may be offered, at each PI's discretion, to any remaining and future patients in this trial. According to clinical trials.gov this study is being conducted under world-renowned Study Director Dr. Rob. ${ }^{5}$

According to Johns Hopkins, doctors and researchers are finding that this coronavirus - officially called severe acute respiratory syndrome coronavirus 2 (SARS-CoV-2) can also cause severe and lasting harm in other organs, including the heart and kidneys. John Sperati, an expert in kidney health, often discusses how the new coronavirus might affect kidney function as the illness develops and afterward as a person recovers. Another clinical article, "ACE2 receptor polymorphism: Susceptibility to SARS-CoV-2, bypertension, multi-organ failure, and COVID-19 disease outcome," Devaux et $\mathrm{al}^{4}$ discusses the relationship between the virus, the peptidase func- 
tion of ACE2 and the levels of angiotensin II in SARS-CoV-2 infected patients should help to better understand the pathophysiology of the disease and the multi-organ failures observed in severe COVID-19 cases, particularly heart failure. The New York Times reported $^{8}$ on multi-organ failure initially on April 18, 2020 when it investigated a surge in COVID-19 patients with kidney failure that is leading to shortages of machines, supplies and staff required for emergency dialysis.

On September $23^{\text {rd }}, 2020$ DURECT dosed the first patient in its randomized, double-blind, placebo-controlled, multi-centre Phase 2 study to evaluate the safety and efficacy of DUR928 in hospitalized COVID-19 patients with acute liver or kidney injury. The primary efficacy endpoint is a composite of survival and being free of acute organ failure at Day 28. Based on the corporate website, the company plans to enrol approximately $80 \mathrm{pa}$ tients in multiple study sites across the US.

\section{CONCLUSION}

This analysis has evaluated two studies in the public domain; Humanigen with its phase 3 randomized, double-blind, multicenter, placebo-controlled clinical trial will evaluate the impact of lenzilumab (anti-human GM-CSF monoclonal antibody) on time to recovery in hospitalized patients with severe or critical COVID-19 pneumonia, and Durect's DUR-928 to evaluate safety and efficacy of DUR-928 in treatment of acute organ failure in subjects infected with SARS-CoV-2. We have established some of the critical thinking involved in expedited timelines, expanded access, and rapidly developing research trends in the biotechnology and CRO space as therapeutics and their IND status was being considered by regulatory bodies. The purpose of this article, therefore, is to clarify the decision-making pattern of biotech companies and CRO's when faced with a global pandemic in order to shed light on the global interventional mechanisms with the pharmaceutical and biotechnology industries undertake in partnership with CRO's.

\section{CONFLICTS OF INTEREST}

The authors declare that they have no conflicts of interest.

\section{REFERENCES}

1. Wu C, Chen X, Cai Y, Xia J, Zhou X, Xu S, et al. Risk factors associated with acute respiratory distress syndrome and death in patients with coronavirus disease 2019 pneumonia in Wuhan, China. JAMA Intern Med. 2020; 180(7): 934-943. doi: 10.1001/jamainternmed.2020.0994

2. Kant S, Menez SP, Hanouneh M, Fine DM, Crews DC, Brennan DC, et al. The Covid-19 nephrology compendium: AKI, CKD, ESKD and transplantation. BMC Nephrol. 2020; 21(1): 449. doi: 10.1186/s12882-020-02112-0

3. Abelson R, Fink S, Kulish N, Thomas K. An overlooked, possibly fatal coronavirus crisis: A dire need for kidney dialysis. The New York Times. Published April 18, 2020. Web site. https://www.nytimes.com/2020/04/18/health/kidney-dialysis-coronavirus.html. Accessed November 22, 2020.

4. Devaux CA, Rolain J-M, Raoult D. ACE2 receptor polymorphism: Susceptibility to SARS-CoV-2, hypertension, multi-organ failure, and COVID-19 disease outcome. J Microbiol Immunol Infect. 2020; 53(3): 425-435. doi: 10.1016/j.jmii.2020.04.015

5. Phase 3 Study to Evaluate Efficacy and Safety of Lenzilumab in Patients With COVID-19. Web site. https://clinicaltrials.gov/ct2/ show/NCT04351152. Accessed November 22, 2020.

6. Rob G. DUR-928 in Subjects With SARS-CoV-2 With Acute Liver or Kidney Injury. Web site. https://clinicaltrials.gov/ct2/ show/NCT04447404. Accessed November 22, 2020.

7. Mohammadi M, Meskini M, do Nascimento Pinto AL. Novel coronavirus (COVID-19) overview. J Public Health (Berl). 2020. doi: 10.1007/s10389-020-01258-3

8. Kim WC, Mauborgne R. Blue ocean strategy: How to create uncontested market space and make the competition irrelevant. Brighton, MA, USA: Harvard Business School Press; 2004. 